\title{
PARAMETER ESTIMATION IN BAYESIAN RECONSTRUCTION OF MULTISPECTRAL IMAGES USING SUPER RESOLUTION TECHNIQUES
}

\author{
Rafael Molina ${ }^{a}$, Miguel Vega ${ }^{b}$, Javier Mateos ${ }^{a}$, and Aggelos K. Katsaggelos ${ }^{c}$
}

a) Dept. Ciencias de la Computación e I. A., Univ. de Granada, 18071 Granada, Spain. b) Dept. de Lenguajes y Sistemas Informáticos, Univ. de Granada, 18071 Granada, Spain.

c) Dept. of Electrical Engineering and Computer Science,

Northwestern University, Evanston, Illinois 60208-3118.

mvega@ugr.es,jmd@decsai.ugr.es,rms@decsai.ugr.es,aggk@ece.northwestern.edu

\begin{abstract}
In this paper we present a new super resolution Bayesian method for pansharpening of multispectral images which: a) incorporates prior knowledge on the expected characteristics of the multispectral images, b) uses the sensor characteristics to model the observation process of both panchromatic and multispectral images, and c) performs the estimation of all the unknown parameters in the model. Using real data, the pansharpened multispectral images are compared with the images obtained by other parsharpening methods and their quality is assessed both qualitatively and quantitatively.
\end{abstract}

Index Terms - Hierarchical Bayesian modeling, super resolution, image reconstruction, pansharpening multispectral images

\section{INTRODUCTION}

Nowadays most remote sensing systems include sensors able to simultaneously capture several low resolution images of the same area on different wavelengths, thus forming a multispectral image, along with a high resolution panchromatic image. For instance, the Landsat 7 satellite, equipped with the ETM+ sensor, allows for the capture of a multispectral image with six bands (three bands in the visible plus three bands in the infrared spectrum) with a resolution of 30 meters per pixel, a thermal band with a resolution of 60 meters per pixel and a panchromatic band (covering a large zone in the visible and the near infrared spectrum), with a resolution of 15 meters per pixel.

In this paper the term multispectral image reconstruction using super resolution techniques will refer to the joint processing of the multispectral and panchromatic images in order to obtain a new multispectral image that, ideally, will present the spectral characteristics of the observed multispectral

This work has been partially supported by the "Comisión Nacional de Ciencia y Tecnología" under contract TIC2003-00880, by the Greece-Spain Integrated Action HG2004-0014, and by the "Instituto de Salud Carlos III" project FIS G03/185. image and the spatial resolution and quality of the panchromatic image.

A few approximations to this problem have been proposed in the literature (see [1] for a comparative study). Among them principal component analysis (PCA) [2] and wavelets based approaches [3] have been proposed. Price [4] proposed a method relying on the statistical relationships between the radiances in the low and high spatial resolution bands. Recently a few super-resolution based methods have also been proposed $[5,6]$.

In this paper we formulate the problem of multispectral image reconstruction using super resolution techniques from a Bayesian perspective and derive a new method to simultaneously estimate the parameters of the model and the high resolution multispectral image from the observed images. The method extends the results already presented in [7], where an iterative algorithm for the reconstruction problem, assuming the values of the parameters were known, was proposed.

The paper is organized as follows. The acquisition model is presented in section 2. In section 3 the Bayesian paradigm for super resolution applied to multispectral image reconstruction is presented and the required probability distributions are formulated. The Bayesian analysis is performed in section 4 to obtain the reconstruction and parameter estimation algorithm. Experimental results on a real Landsat 7 ETM+ image are described in section 5. Finally, section 6 concludes the paper.

\section{ACQUISITION MODEL}

Let us assume that $\mathbf{y}$, the multispectral image we would observe under ideal conditions with a high resolution sensor, has $B$ bands, that is, $\mathbf{y}=\left[\mathbf{y}_{1}^{t}, \mathbf{y}_{2}^{t}, \ldots, \mathbf{y}_{B}^{t}\right]^{t}$, where each one of the bands is of size $p=m \times n$ pixels, and $t$ denotes the transpose of a vector or matrix.

Each band of this image can be expressed as a column vector by lexicographically ordering the pixels in the band as $\mathbf{y}_{b}=\left[y_{b}(1,1), y_{b}(1,2), \ldots, y_{b}(m, n)\right]^{t}, b=1,2, \ldots, B$. 
In real applications, however, this multispectral image is not available and, instead, we observe a low resolution multispectral image $\mathbf{Y}$ with $B$ bands $\mathbf{Y}=\left[\mathbf{Y}_{1}^{t}, \mathbf{Y}_{2}^{t}, \ldots, \mathbf{Y}_{B}^{t}\right]^{t}$, where each low resolution image is of size $P=M \times N$ pixels, with $M<m$ and $N<n$.

Using the previously described ordering we have $\mathbf{Y}_{b}=$ $\left[Y_{b}(1,1), Y_{b}(1,2), \ldots, Y_{b}(M, N)\right]^{t}$, for $b=1,2, \ldots, B$. Each band, $\mathbf{Y}_{b}$, is related to its corresponding high resolution image by

$$
\mathbf{Y}_{b}=\mathbf{H y}_{b}+\mathbf{n}_{b}, \quad \forall b=1, \cdots, B,
$$

where $\mathbf{H}$ is a $P \times p$ matrix representing the blurring, the sensor integration function and the spatial subsampling (we assume that this process is the same for the whole set of spectral images) and $\mathbf{n}_{b}$ is the capture noise, assumed to be Gaussian with zero mean and variance $1 / \beta_{b}$.

A simple but widely used model for the matrix $\mathbf{H}$ is to consider that each pixel $(i, j)$ of the low resolution image is obtained according to (for $m=2 M$ and $n=2 N$ )

$$
Y_{b}(i, j)=\frac{1}{4} \sum_{(u, v) \in E_{i, j}} y_{b}(u, v)+n_{b}(i, j),
$$

where $E_{i, j}$ consists of the indices of the four high resolution pixels $\{(2 i, 2 j),(2 i+1,2 j),(2 i, 2 j+1),(2 i+1,2 j+1)\}$.

We note here that $\mathbf{H}$ can be written as

$$
\mathbf{H}=\mathbf{D B} \text {, }
$$

where $\mathbf{B}$ is a $p \times p$ blurring matrix and $\mathbf{D}$ is a decimation operator.

The sensor also provides us with a panchromatic image $\mathbf{x}$ of size $p=m \times n$, obtained by spectral averaging the unknown high resolution images $\mathbf{y}_{b}$. This relation can be modelled as

$$
\mathbf{x}=\sum_{b=1}^{B} \lambda_{b} \mathbf{y}_{b}+\nu
$$

where $\lambda_{b} \geq 0, b=1,2, \cdots, B$, weight the contribution of each band $\mathbf{y}_{b}$, based on the sensor spectral response (see Fig. 1), to the panchromatic image $\mathbf{x}$ and $\nu$ is the capture noise that is assumed to be Gaussian with zero mean and variance $\gamma^{-1}$.

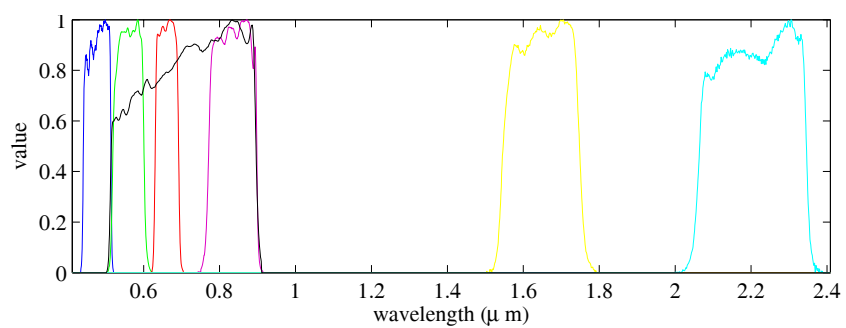

Fig. 1. Normalized Landsat 7 ETM+ band spectral responses.

\section{BAYESIAN PARADIGM}

We aim at reconstructing the multispectral high resolution image $\mathbf{y}$ from the observed multispectral low resolution image $\mathbf{Y}$ and the high resolution panchromatic image $\mathbf{x}$. Following the Bayesian paradigm we have to formulate the observation and prior models.

\subsection{Degradation model}

It can be assumed that $\mathbf{Y}$ and $\mathbf{x}$, that are obtained by distinct sensors, are independent for a given $\mathbf{y}$ and consequently ${ }^{1}$

$$
\mathrm{p}(\mathbf{Y}, \mathbf{x} \mid \mathbf{y})=\mathrm{p}(\mathbf{Y} \mid \mathbf{y}) \mathrm{p}(\mathbf{x} \mid \mathbf{y}) .
$$

Given the degradation model for multispectral image super resolution described by Eq. (1) the distribution of the observed $\mathbf{Y}$ given $\mathbf{y}$ is given by

$$
\mathrm{p}(\mathbf{Y} \mid \mathbf{y})=\prod_{b=1}^{B}\left(\frac{\beta_{b}}{2 \pi}\right)^{P / 2} \exp \left\{-\frac{1}{2} \sum_{b=1}^{B} \beta_{b}\left\|\mathbf{Y}_{b}-\mathbf{H y}_{b}\right\|^{2}\right\} .
$$

Using the degradation model in Eq. (4), the distribution of the panchromatic image $\mathbf{x}$ given $\mathbf{y}$ is given by

$$
\mathrm{p}(\mathbf{x} \mid \mathbf{y})=\left(\frac{\gamma}{2 \pi}\right)^{p / 2} \exp \left\{-\frac{1}{2} \gamma\left\|\mathbf{x}-\sum_{j=1}^{B} \lambda_{j} \mathbf{y}_{j}\right\|^{2}\right\} .
$$

\subsection{Image model}

It is also necessary to specify the prior distribution of the high resolution image $\mathrm{p}(\mathbf{y})$. Although other models are possible, in this paper we assume no correlation between the different high resolution bands. Then, our prior knowledge about the smoothness of the object luminosity distribution within each band makes it possible to model the distribution of $\mathbf{y}_{b}$ by

$$
\mathrm{p}(\mathbf{y})=\prod_{b=1}^{B} \mathrm{p}\left(\mathbf{y}_{b}\right)=\prod_{b=1}^{B}\left(\frac{\alpha_{b}}{2 \pi}\right)^{p / 2} \prod_{b=1}^{B} \exp \left\{-\frac{1}{2} \alpha_{b}\left\|\mathbf{C y}_{b}\right\|^{2}\right\},
$$

where $\mathbf{C}$ denotes the Laplacian operator, $1 / \alpha_{b}$ is the variance of the Gaussian distribution.

Note that from the above definitions of the probability density functions, the distribution

$$
\mathrm{p}(\mathbf{y}, \mathbf{Y}, \mathbf{x})=\mathrm{p}(\mathbf{y}) \mathrm{p}(\mathbf{Y} \mid \mathbf{y}) \mathrm{p}(\mathbf{x} \mid \mathbf{y})
$$

depends on a set of unknown parameters and has to be properly written as $\mathrm{p}(\mathbf{y}, \mathbf{Y}, \mathbf{x} ; \Theta)$, where

$$
\Theta=\left(\alpha_{1}, \alpha_{2}, \ldots, \alpha_{B}, \beta_{1}, \beta_{2}, \ldots, \beta_{B}, \gamma\right) .
$$

\footnotetext{
${ }^{1}$ If $\mathbf{Y}$ and $\mathbf{x}$ were not independent, $\mathrm{p}(\mathbf{Y}, \mathbf{x} \mid \mathbf{y})=\mathrm{p}(\mathbf{Y} \mid \mathbf{y}) \mathrm{p}(\mathbf{x} \mid \mathbf{Y}, \mathbf{y})$ should be written.
} 


\section{BAYESIAN INFERENCE}

In this paper we use maximum likelihood for estimating the parameters, $\Theta$, and maximum a posteriori for estimating the high-resolution multispectral image $y$. Towards that, the following two steps are required.

\section{Step I: Estimation of $\Theta$}

$$
\begin{aligned}
& \text { Find } \hat{\Theta}=\left(\hat{\alpha}_{1}, \hat{\alpha}_{2}, \ldots, \hat{\alpha}_{B}, \hat{\beta}_{1}, \hat{\beta}_{2}, \ldots, \hat{\beta}_{B}, \hat{\gamma}\right) \text { as } \\
& \hat{\Theta}=\arg \max _{\Theta} \log \int_{\mathbf{y}} \mathrm{p}(\mathbf{y}, \mathbf{Y}, \mathbf{x} ; \Theta) d \mathbf{y}
\end{aligned}
$$

\section{Step II: Estimation of $y$}

Choose $\mathbf{y}(\Theta)$ as

$$
\mathbf{y}(\Theta)=\arg \max _{\mathbf{y}} \log \mathrm{p}(\mathbf{y} \mid \mathbf{Y}, \mathbf{x} ; \Theta) .
$$

Then, once $\Theta=\hat{\Theta}$ has been selected in the step I above the estimation of $\mathbf{y}$ is found by using $\Theta=\hat{\Theta}$ in Eq. (12).

In order to estimate $\Theta$ we now apply the E-M algorithm [8]. Calculating

$$
\mathbf{U}\left(\Theta \mid \Theta^{\prime}\right)=\mathbf{E}_{\mathbf{y} \mid \mathbf{Y}, \mathbf{x} ; \Theta^{\prime}}[\log \mathrm{p}(\mathbf{y}, \mathbf{x}, \mathbf{Y} ; \Theta)] .
$$

and differentiating $\mathbf{U}\left(\Theta \mid \Theta^{\prime}\right)$ with respect to $\Theta$ we obtain at its maximum

$$
\begin{aligned}
\frac{p}{\alpha_{b}}= & \left\|\mathbf{C y}_{b}\left(\Theta^{\prime}\right)\right\|^{2}+\operatorname{tr}\left[\mathbf{C}^{t} \mathbf{C} \operatorname{cov}\left(\mathbf{y}_{b} \mid \mathbf{Y}, \mathbf{x} ; \Theta^{\prime}\right)\right] \\
& b=1, \ldots, B, \\
\frac{P}{\beta_{b}}= & \left\|\mathbf{Y}_{b}-\mathbf{H y}_{b}\left(\Theta^{\prime}\right)\right\|^{2}+\operatorname{tr}\left[\mathbf{H}^{t} \mathbf{H} \mathbf{c o v}\left(\mathbf{y}_{b} \mid \mathbf{Y}, \mathbf{x} ; \Theta^{\prime}\right)\right] \\
& b=1, \ldots, B, \\
\frac{p}{\gamma}= & \left\|\mathbf{x}-\sum_{b=1}^{B} \lambda_{b} \mathbf{y}_{b}\left(\Theta^{\prime}\right)\right\|^{2} \\
+ & \sum_{i=1}^{B} \sum_{j=1}^{B} \lambda_{i} \lambda_{j} \operatorname{tr}\left[\mathbf{c o v}\left(\mathbf{y}_{i}, \mathbf{y}_{j} \mid \mathbf{Y}, \mathbf{x} ; \Theta^{\prime}\right)\right] .
\end{aligned}
$$

We will use the following algorithm for the estimation of the hyperparameters and the high-resolution image

Algorithm 1 Iterative estimation of $\hat{\Theta}$ and $\mathbf{y}(\hat{\Theta})$.

1. Choose $\Theta^{0}$. Set $k=0$.

2. Compute $\mathbf{y}\left(\Theta^{0}\right)$ using Eq. (12) for $\Theta=\Theta^{0}$.

3. Repeat

i) Set $k=k+1$

ii) Use $\Theta^{\prime}=\Theta^{k-1}$ in the right hand side of Eqs. (14), (15) and (16) to obtain $\Theta^{k}$ in the left hand side of these equations. iii) Compute $\mathbf{y}\left(\Theta^{k}\right)$ using Eq. (12) for $\Theta=\Theta^{k}$

until

$$
\frac{\left\|\mathbf{y}\left(\Theta^{k}\right)-\mathbf{y}\left(\Theta^{k-1}\right)\right\|^{2}}{\left\|\mathbf{y}\left(\Theta^{k}\right)\right\|^{2}}<\epsilon
$$

where $\epsilon$ is prescribed bound.

It can be shown that all calculations needed to find the parameters solution of Eq. (11) and the multispectral image solution of Eq. (12) can be performed in an efficient way in the frequency domain by extending to multichannel processing the framework described in [9]. Eq. (12) has been solved analytically, while in [7] an iterative algorithm was applied.

\section{EXPERIMENTAL RESULTS}

Algorithm 1 has been tested on the Landsat 7 ETM+ image set [10] of Table 1. Multispectral image zones of $256 \times 256$ pixels, and their corresponding $512 \times 512$ pixel panchromatic images, were used in the experiment.

\begin{tabular}{|c|c|c|c|}
\hline Image & Date & Path & Row \\
\hline$A$ & $2000-08-08$ & 199 & 031 \\
\hline$B$ & $2000-07-30$ & 200 & 031 \\
\hline
\end{tabular}

Table 1. Landsat 7 ETM+, L1G Orthorectified image sets.

Taking into account the ETM+ band spectral responses in Fig. 1 we have applied our method to the first $B=4$ bands of the images. The values of $\lambda_{b}, b=1, \ldots, 4$, have been obtained by summing up the panchromatic sensor response, weighted by the response of each band sensor, and normalizing them to sum up to one, giving $\left[\lambda_{1}, \lambda_{2}, \lambda_{3}, \lambda_{4}\right]=[0.0078$, $0.2420,0.2239,0.5263]$.

In all experiments the proposed Algorithm 1 was run using $\gamma^{0}=1.0$, and $\alpha_{b}^{0}=0.01$, and $\beta_{b}^{0}=100.0$, for $b=$ $1, \ldots, 4$, as initial values. The value $\epsilon=10^{-6}$ was used in Eq. (17) as upper bound. In all that follows, table and figure legends included, the different methods under comparison are referenced as: MI Bicubic interpolation, MII Price method [4], MIII Proposed method.

Figure 2 shows a detail of the reconstructions of image $A$ using MI, MII and MIII. Table 2 shows a numerical comparison of the different reconstruction methods in terms of $\mathrm{PSNR}_{b}=10 \log _{10}\left\{\frac{255}{\left\|\mathbf{Y}_{b}-\mathbf{D y}_{b}\right\|}\right\}$. MIII outperforms MI and MII, both in quantitative and qualitative terms.

A second experiment, in which the observation process was simulated, was performed. Simulated observed multispectral $128 \times 128$ images were generated by applying sensor integration and downsampling to the original images. Table 3 shows a numerical comparison of the different reconstruction methods both in terms of the UIQI index and $\Delta_{S N R}$ in $\mathrm{dB}$. UIQI, proposed and well described in [11], is an overall image quality index that takes into account the degree of correlation, 


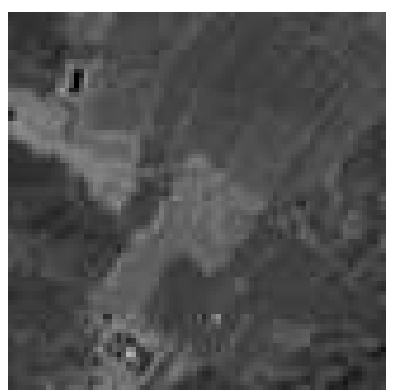

(a)

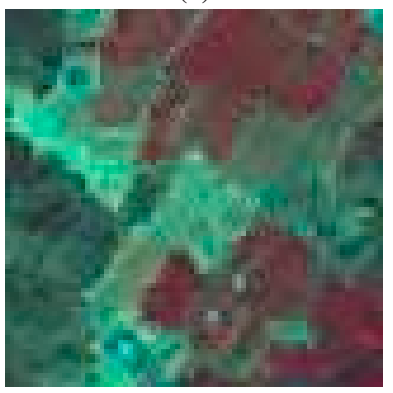

(c)

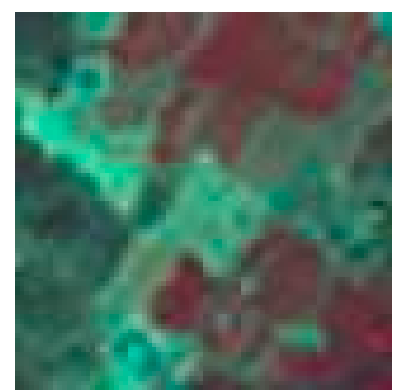

(b)

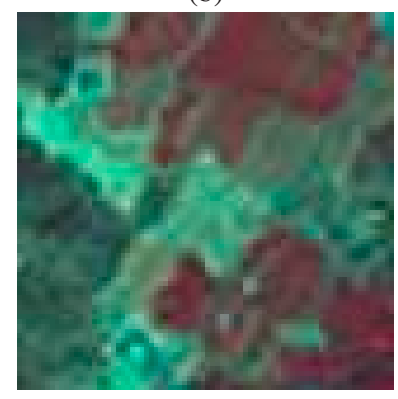

(d)
Fig. 2. (a) Details of the panchromatic image $A$ in Table 1 and the $\operatorname{RGB}\left(\hat{\mathbf{y}}_{4}, \hat{\mathbf{y}}_{3}, \hat{\mathbf{y}}_{2}\right)$ reconstruction using (b) MI, (c) MII, and (d) MIII.

\begin{tabular}{|c|c|c|c|c|}
\hline Image & band & MI & MII & MIII \\
\hline \multirow{3}{*}{$A$} & 1 & 19.1 & 17.7 & 19.5 \\
\cline { 2 - 5 } & 2 & 17.9 & 16.5 & 18.0 \\
\cline { 2 - 5 } & 3 & 15.8 & 14.4 & 15.9 \\
\cline { 2 - 5 } & 4 & 17.8 & 16.7 & 17.9 \\
\hline$B$ & 1 & 20.1 & 18.4 & 20.9 \\
\cline { 2 - 5 } & 2 & 18.9 & 17.4 & 19.7 \\
\cline { 2 - 5 } & 3 & 16.3 & 14.8 & 17.1 \\
\cline { 2 - 5 } & 4 & 19.8 & 17.7 & 19.6 \\
\hline
\end{tabular}

Table 2. PSNR (dB) obtained in the first experiment.

the closeness of the luminance and the similarity of the contrast of the images under comparison. UIQI takes values in the range $[-1,1]$. The larger the value of the index, the better the quality of the image. Again, MIII outperforms MI and MII, obtaining a higher UIQI and better $\Delta_{S N R}$.

\section{CONCLUSIONS}

In this paper the reconstruction of multispectral images has been formulated from a superresolution point of view. A new method for estimating both the reconstructed pansharpened images and the model parameters, within the Bayesian framework, was proposed. Based on the presented experimental results, the new method outperform bicubic interpolation and the method in [4].

\section{REFERENCES}

[1] V. Vijayaraj, "A quantitative analysis of pansharpened images," M.S. thesis, Mississippi St. Univ., 2004.

[2] P. Chavez Jr., S. Sides, and J. Anderson, "Comparison

\begin{tabular}{|c|c|c|c|c|c|c|c|}
\hline & & \multicolumn{3}{|c|}{ UIQI } & \multicolumn{3}{|c|}{$\Delta_{S N R}$} \\
\hline Image & band & MI & MII & MIII & MI & MII & MIII \\
\hline \multirow{4}{*}{$A$} & 1 & 0.76 & 0.78 & 0.83 & 24.6 & 23.5 & 26.9 \\
\cline { 2 - 8 } & 2 & 0.79 & 0.79 & 0.88 & 21.6 & 20.6 & 24.9 \\
\cline { 2 - 8 } & 3 & 0.79 & 0.80 & 0.88 & 18.8 & 17.8 & 22.1 \\
\cline { 2 - 8 } & 4 & 0.80 & 0.80 & 0.90 & 21.1 & 20.0 & 24.8 \\
\hline \multirow{4}{*}{$B$} & 1 & 0.71 & 0.67 & 0.78 & 24.9 & 22.8 & 27.0 \\
\cline { 2 - 8 } & 2 & 0.76 & 0.69 & 0.83 & 21.9 & 19.8 & 24.8 \\
\cline { 2 - 8 } & 3 & 0.76 & 0.70 & 0.83 & 18.1 & 15.9 & 21.0 \\
\cline { 2 - 7 } & 4 & 0.75 & 0.68 & 0.82 & 24.9 & 22.5 & 27.5 \\
\hline
\end{tabular}

Table 3. UIQI and $\Delta_{S N R}(\mathrm{~dB})$ obtained in the second experiment.

of three different methods to merge multiresolution and multispectral data: Landsat TM and SPOT panchromatic," Phot. Eng. \& Rem. Sens., vol. 57, no. 3, pp. 295303, 1991.

[3] G. Pajares and J. M. de la Cruz., "A wavelet-based image fusion tutorial," Pattern Recognition, vol. 37, pp. 1855-1872, 2004.

[4] J.C. Price, "Combining multispectral data of different spatial resolution," IEEE Trans. on Geosc. \& Rem. Sens., vol. 37, no. 3, pp. 1199-1203, 1999.

[5] M.T. Eismann and R.C. Hardie, "Hyperspectral resolution enhancement using high-resolution multispectral imaginary with arbitray response functions," IEEE Trans. on Geosc. \& Rem. Sens., vol. 43, no. 3, pp. 455465, 2005.

[6] T. Akgun, Y. Altunbasak, and R.M. Mersereau, "Superresolution reconstruction of hyperspectral images," IEEE Trans. on Img. Proc., vol. 14, no. 11, pp. 18601875, 2005.

[7] R. Molina, J. Mateos, A.K. Katsaggelos, and R. ZuritaMilla, "A new super resolution Bayesian method for pansharpening landsat ETM+ imagery," in 9th Int. Sym. on Phys. Meas. and Signatures in Rem. Sens. (ISPMSRS), S. Liang, J. Liu, X. Li, R. Liu, and M. Schaepman, Eds. Beijing (China), Oct 2005, pp. 280-283.

[8] A. D. Dempster, N. M. Laird, and D. B. Rubin, "Maximum likelihood from incomplete data via the E-M algorithm," J. Roy. Stat. Soc. B, vol. 39, pp. 1-37, 1977.

[9] R. Molina, M. Vega, J. Abad, and A.K. Katsaggelos, "Parameter estimation in Bayesian high-resolution image reconstruction with multisensors," IEEE Trans. on Img. Proc., vol. 12, no. 12, pp. 1655-1667, 2003.

[10] NASA Landsat Program, "Landsat ETM+ Scenes," in Global Land Cover Facility. U.S. Geological Survey, Sioux Falls, South Dakota.

[11] Z. Wang and A.C. Bovik, "A universal image quality index,” IEEE Sign. Proc. Lett., vol. 9, pp. 81-84, 2002. 